\title{
Using of machine parts abstract elements in nc- programs developing for the cnc machines
}

\author{
Mihail Nazarov ${ }^{1}$, Evgeny Kiselev ${ }^{1, *}$, and Aleksey Popovich ${ }^{1}$ \\ ${ }^{1}$ Ulyanovsk State Technical University 32, Severny Venetz str.,432027 Ulyanovsk, Russia
}

\begin{abstract}
Described questions of automation for the process-layout preparation with using of workpieces abstract elements in $\mathrm{NC}$ programming. Presented views of various elements parts workpieces and algorithms of functioning of the automated CAM-system.
\end{abstract}

In process-layout preparation - it is necessary to minimization of the possible errors linked with the human factor and capable to cause failures during parts machining.

In modern CAM's, there are a large number of algorithms that allow to semi-automate a design process and toolpath generation for any types of machining parts (a boss, a pocket, a surface and a hole), but this automation possibilities is not enough for implementation exchange of machining experience from users groups. Because many differences in algorithms for processing the above elements are now available. One of solution for this question can be a general algorithm for processing all elements that will operate with parameters for processing with the use of only small adjustments to adapt the trajectories to different types of elements.

For example, machining of the pocket and the boss will be a little bit other if you slightly change the geometric representation of the boss.

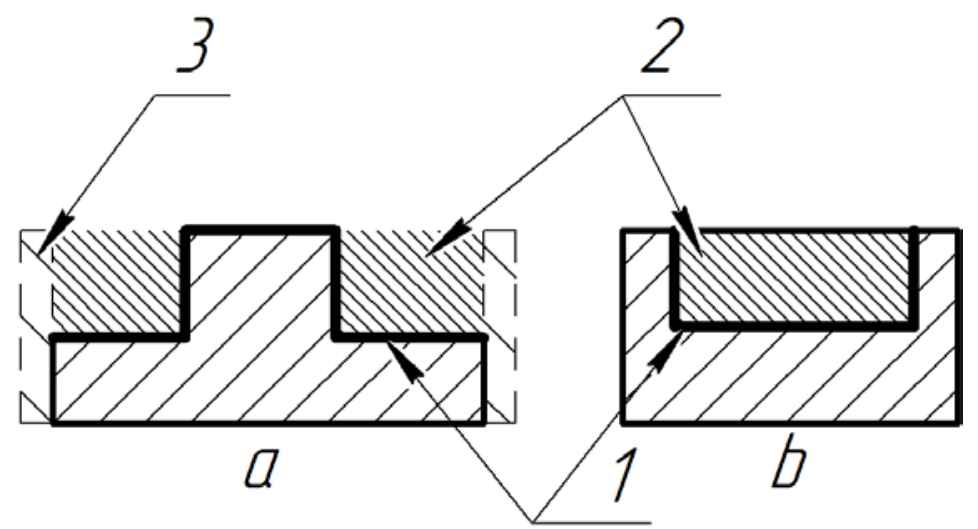

Fig. 1. The boss machining through the pocket representation

\footnotetext{
${ }^{*}$ Corresponding author: kec.ulstu@mail.ru
} 
The figure shows two elements of the part: $\mathrm{a}$ - is a boss, $\mathrm{b}$ - is a pocket, one is a machining's surfaces, second is a removed material after machining. A boss machining can be present as a pocket machining if you add virtual walls on the surface boundaries of the adjacent to a boss. Thus, we get a pocket with an island inside, which have only little differ in machining - the method of tool plunging (from the outside - "air", but not into part material). In this case, we operate with parameters for both cases: removed part material, contour length, machining element depth and height, fillet radius for the contour corners. After this additional work, it is possible to automate the machining both of these elements by one algorithm, only with some approaching cutting method correction.

Following this algorithm, the CAM, for example, Siemens NX, with additional modules is able to determine the necessary set of input parameters in automatic mode, for their subsequent handling on the server side and selection of a workable set of technological parameters. After visualization and confirmation, the system generates trajectories for moving the cutting tool and outputs them through the postprocessor to the metal cutting equipment.

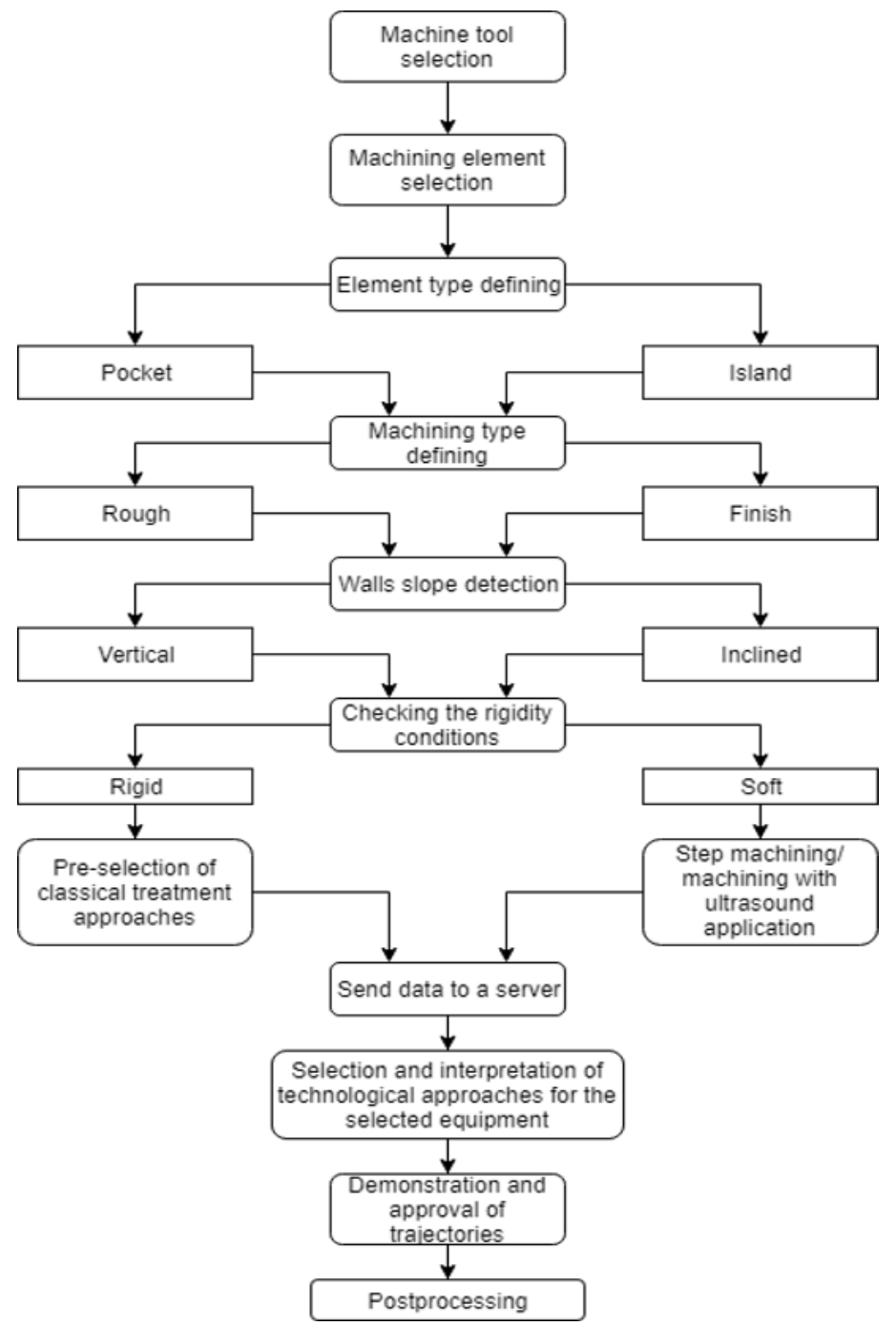

Fig. 2. Elements and machining recognition algorithm. 
Machining of the elements is carried out according to the algorithm given below, with a check of a number of conditions that determine the stages necessary for execution and the stages, the execution of which is not necessary.

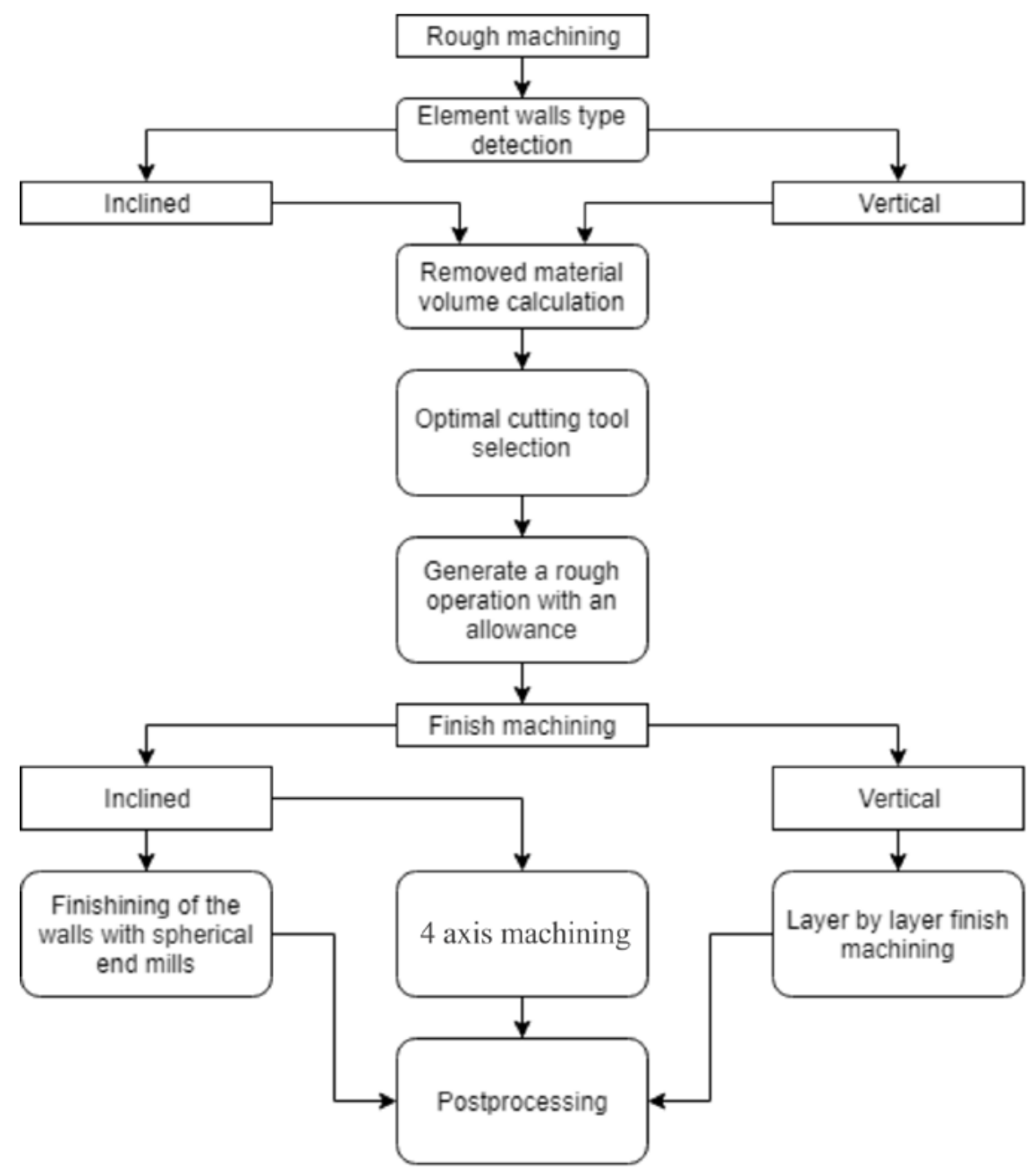

Fig. 3. Sequence of actions for the machining operations generation.

The system, based on these algorithms, can be integrated into any standalone or online (working directly in internet browser) CAM software. The server makes all calculations, without requiring large computing calculation power from the user, besides there is no need to create an interaction between the client application of the CAM software and its server computing part. All solutions of the online system are accepted immediately based on the experience of user groups, which is constantly collected and updated.

Thus, a number of the solutions proposed above will result in almost complete automation of the creation of machining operations, which takes into account the type of equipment, starting from old $\mathrm{CNC}$ machines, ending with the most modern machining centers equipped with ultrasonic technologies for improve a cutting tools possibilities during part machining (for example, a machines from DMG-MORI - Ultrasonic series).

Researches was carried out as part of grant works by 16-47-732010 r_ofi_m RFBR grant. 


\section{References}

1. M.V. Nazarov, A.V. Popovich, E.S. Kiselev, Automated choice of manufacturing techniques of aircraft details, Izvestiya TulGU, (8), pp. 147-153 (2017)

2. E.S. Kiselev, S.A. Imandinov, M.V. Nazarov, Quality assurance features non-rigid aluminum blanks when milling with ultrasonic vibrations, Izvestiya VolgGTU (12), pp. 14-17 (2017) 